\title{
Chiral symmetry and quantum hadrodynamics
}

\author{
G. Chanfray, ${ }^{1}$ M. Ericson, ${ }^{1,2}$ and P. A. M. Guichon ${ }^{3}$ \\ ${ }^{1}$ IPN Lyon, IN2P3-CNRS et UCB Lyon I, F-69622 Villeurbanne Cedex, France \\ ${ }^{2}$ Theory Division, CERN, CH-12111 Geneva, Switzerland \\ ${ }^{3}$ SPhN/DAPNIA, CEA-Saclay, F-91191 Gif sur Yvette Cedex, France
}

(Received 5 December 2000; published 4 April 2001)

\begin{abstract}
Using the linear sigma model, we study the evolutions of the quark condensate and of the nucleon mass in the nuclear medium. Our formulation of the model allows the inclusion of both pion and scalar-isoscalar degrees of freedom. It guarantees that the low-energy theorems and the constraints of chiral perturbation theory are respected. We show how this formalism incorporates quantum hadrodynamics improved by the pion loop effects.
\end{abstract}

\section{INTRODUCTION}

The influence of the nuclear medium on the spontaneous breaking of chiral symmetry remains an open problem. The amount of symmetry breaking is measured by the quark condensate, which is the expectation value of the quark operator $\bar{q} q$. The vacuum value $\langle\bar{q} q(0)\rangle$ satisfies the Gell-Mann, Oakes, and Renner relation

$$
2 m_{q}\langle\bar{q} q(0)\rangle=-m_{\pi}^{2} f_{\pi}^{2},
$$

where $m_{q}$ is the current quark mass, $q$ the quark field, $f_{\pi}$ $=93 \mathrm{MeV}$ the pion decay constant, and $m_{\pi}$ its mass. In the nuclear medium the quark condensate decreases in magnitude. Indeed the total amount of restoration is governed by a known quantity, the nucleon sigma commutator $\Sigma_{N}$ which, for any hadron $h$ is defined as

$$
\begin{aligned}
\Sigma_{h} & =-i\left\langle h\left|\left[Q_{5}, \dot{Q}_{5}\right]\right| h\right\rangle \\
& =2 m_{q} \int d \vec{x}[\langle h|\bar{q} q(\vec{x})| h\rangle-\langle\bar{q} q(0)\rangle],
\end{aligned}
$$

where $Q_{5}$ is the axial charge and $\dot{Q}_{5}$ its time derivative. At low density, where the nucleons do not interact, one can estimate the restoration effect by adding the contributions of the individual nucleons. This leads to $[1,2]$

$$
\frac{\langle\bar{q} q(\rho)\rangle}{\langle\bar{q} q(0)\rangle}=1-\frac{\Sigma_{N} \rho}{m_{\pi}^{2} f_{\pi}^{2}},
$$

where $\rho$ is the density. Using the experimental value $\Sigma_{N}$ $\propto 50 \mathrm{MeV}$ one thus gets a relative drop of almost $40 \%$ at normal density $\rho_{0}=0.17 \mathrm{fm}^{-3}$.

The part of the restoration process which is best understood arises from the nuclear virtual pions. They act in the same way as the real ones of the heat bath, leading to a similar expression for the evolution of the quark condensate, that is,

$$
\frac{\langle\bar{q} q(\rho, T)\rangle}{\langle\bar{q} q(0,0)\rangle}=1-\frac{\rho_{s}^{\pi}(\rho, T)}{2 m_{\pi} f_{\pi}^{2}}=1-\frac{\left\langle\phi^{2}\right\rangle}{2 f_{\pi}^{2}},
$$

where $T$ is the temperature and the scalar pion density $\rho_{s}^{\pi}$ is linked to the average value of the squared pion field $\phi^{2}$ $=\vec{\phi} \cdot \vec{\phi}$ through (it is understood that the vacuum contribution to $\left\langle\phi^{2}\right\rangle$ is substracted)

$$
\rho_{s}^{\pi}=m_{\pi}\left\langle\phi^{2}\right\rangle
$$

Estimates of the right-hand side (RHS) of Eq. (5) for nuclear matter at normal density give $\rho_{s}^{\pi} \propto 0.07 \mathrm{fm}^{-3}$ which leads to a $20 \%$ relative decrease, due to the pion cloud, of the quark condensate. So half of the restoration is due to the nuclear pion cloud. Concerning the manifestation of the symmetry restoration, the pion cloud produces a correlator mixing effect first introduced in the framework of the heat bath by Dey et al. [3] and adapted to the nuclear case by Chanfray et al. [4].

The restoration of nonpionic origin is by contrast not so well understood. In this paper we clarify the role of the meson clouds with special emphasis on the scalar-isoscalar meson which enters in the relativistic models of nuclei. It is expected to play a distinguished role since it has the same quantum numbers as the condensate and can thus dissolve into it. At variance with the scalar-isoscalar meson, which contributes already at the mean field level, the other mesons, including the pion, contribute only through the fluctuations. On the other hand the scalar-isoscalar meson is also an essential actor of the nuclear dynamics since it provides the medium range attraction which binds the nucleons together in the nucleus. In particular the existence of a scalar field is a central ingredient of quantum hadrodynamics (QHD) $[5,6]$.

The mean scalar field is responsible for the lowering of the nucleon mass $\left(M^{*}\right)$ in the nucleus. Effective values of $M^{*}$ lower than the free mass by several hundreds of $\mathrm{MeV}$ are commonly discussed in QHD. It is quite appealing to interpret this mass reduction as a signal for the symmetry restoration. Indeed one scenario for the Wigner realization of the symmetry is the vanishing of hadronic masses. Partial restoration would then show up as a reduction of the masses. This was the suggestion of Brown and Rho [7] who proposed a scaling law linking the mass reduction to the condensate evolution according to 


$$
\frac{M^{*}}{M}=\frac{f_{\pi}^{*}}{f_{\pi}}=\left[\frac{\langle\bar{q} q(\rho)\rangle}{\langle\bar{q} q(0)\rangle}\right]^{\alpha}, \quad \text { with } \quad \alpha=1 / 3
$$

Birse $[8,9]$ pointed out the difficulties inherent to the scaling law (6). The condensate evolution is, as we have seen previously, partly governed by the expectation value $\left\langle\phi^{2}\right\rangle$ which contains a term of order $m_{\pi}$. It is linked to the nonanalytical part of order $m_{\pi}^{3}$ of the pionic part of the nucleon sigma commutator $\Sigma_{N}$ through the relation

$$
\Sigma_{N}(\text { pionic })=\frac{m_{\pi}^{2}}{2} \int d \vec{x}\left\langle N\left|\phi^{2}(\vec{x})\right| N\right\rangle .
$$

If the mass evolution were to follow the condensate one according to Eq. (6), it would thus contain a term of order $m_{\pi}$, which is forbidden by chiral perturbation theory $[8,9]$. It is thus clear that $\left\langle\phi^{2}\right\rangle$, i.e., the condensate evolution of pionic origin, cannot influence the mass.

This argument, however, does not imply that other actors of the restoration cannot affect the mass. In fact the picture which naturally emerges from the previous discussion is that different components of the restoration may produce different signals. One of them is the axial-vector mixing induced by the pionic type of restoration. Another signal may be the hadron mass reduction and its link to the condensate evolution is studied in the following.

The model we use for this study is the linear sigma model which possesses chiral symmetry and considers the sigma and the pion as chiral partners. We first point out that this model faces a potential problem. In the tree approximation, the nucleon mass has its origin in the spontaneous breaking of the chiral symmetry and is proportional to the condensate. Its in-medium value then follows the condensate evolution. However the tree approximation is not sufficient to describe this evolution which is largely influenced by the pion loops, as discussed previously. If the proportionality between the mass and the condensate evolutions still holds once pion loops are included, then the mass would be affected by the pion loops in the same way as the condensate. But, as explained before, this is forbidden by chiral perturbation theory.

We propose to clarify this point through a reformulation of the linear sigma model. As is well known the predictions of the linear sigma model generally involve cancellations between several graphs. One example is the $\pi N$ scattering amplitude where the contribution from sigma exchange, which by itself violates the soft pion results, combines with the Born term to satisfy them. We will see that this is also the case in the mass evolution problem. Namely, the mass evolution, even though it is linked to the condensate evolution, is independent of the pion density.

To mention some previous works, Birse and McGovern [10] investigated the evolution of the condensate with the density up to second order. This was done in the usual formulation of the linear sigma model and pion loops were not included. On the other hand Delorme et al. [11] performed a similar investigation in the nonlinear sigma model which is well adapted for the pion loops but ignores the role of the scalar meson exchange. Our formulation allows one to include both effects, which is necessary in order to discuss the relation between the mass and condensate evolutions.

Our article is organized as follows. In Sec. II we remind the reader of the steps which lead from the linear sigma model to the nonlinear one and we present arguments in favor of an alternative formulation. In Sec. III, we reformulate the linear sigma model in the standard nonlinear form for what concerns the pion field but we keep explicitly a scalar degree of freedom (called $\theta$ ) corresponding to the fluctuation along the chiral radius. The resulting form of the Lagrangian automatically embodies the cancellations imposed by chiral symmetry. We tentatively identify this fluctuation with the scalar meson which produces the nucleon nucleon attraction. In Sec. IV we discuss the prediction of this model for the behavior of various in-medium quantities and we make explicit the link with QHD. Section V is our conclusion.

\section{REMINDER OF THE SIGMA MODEL}

The starting point is the usual linear sigma model [12] which is defined by the Lagrangian

$$
\begin{aligned}
\mathcal{L}= & i \bar{\psi} \gamma^{\mu} \partial_{\mu} \psi+\frac{1}{2}\left(\partial_{\mu} \sigma \partial^{\mu} \sigma+\partial_{\mu} \vec{\pi} \cdot \partial^{\mu} \vec{\pi}\right) \\
& +g_{0} \bar{\psi}\left(\sigma+i \vec{\tau} \cdot \vec{\pi} \gamma_{5}\right) \psi-V_{\mathrm{pot}}\left(\sigma^{2}+\pi^{2}\right)+c \sigma
\end{aligned}
$$

where $(\psi, \sigma, \vec{\pi})$ are, respectively, the nucleon, sigma, and pion fields, the arrow indicating the isovector character of the pion. For the meson potential $V_{\text {pot }}$ we take the usual form $V_{\text {pot }}(x)=\lambda\left(x-v^{2}\right)^{2} / 4$.

For later use it is convenient to write $\mathcal{L}$ in terms of the $2 \times 2$ matrix $W=\sigma+i \vec{\tau} \cdot \vec{\pi}$ acting in the nucleon isospin space. Noting $P^{R / L}=\left(1 \pm \gamma_{5}\right) / 2$ the chirality projectors, one can write

$$
\begin{gathered}
\mathcal{L}=\mathcal{L}_{0}+\mathcal{L}_{\chi S B}, \\
\mathcal{L}_{0}=i \bar{\psi} \gamma^{\mu} \partial_{\mu} \psi+g_{0} \bar{\psi}\left[W P^{R}+W^{\dagger} P^{L}\right] \psi \\
+\frac{1}{4} \operatorname{Tr} \partial_{\mu} W \partial^{\mu} W^{\dagger}-\frac{\lambda}{4}\left(\frac{1}{2} \operatorname{Tr} W W^{\dagger}-v^{2}\right)^{2}, \\
\mathcal{L}_{\chi S B}=c \sigma=\frac{c}{2} \operatorname{Tr} W .
\end{gathered}
$$

In this form it is apparent that $\mathcal{L}_{0}$ is invariant under the transformations

$$
P^{R} \psi \rightarrow g_{R} P^{R} \psi, \quad P^{L} \psi \rightarrow g_{L} P^{L} \psi, \quad W \rightarrow g_{L} W g_{R}^{\dagger},
$$

where $\left(g_{R}, g_{L}\right)$ are elements of the $\mathrm{SU}(2) \times \mathrm{SU}(2)$ group. The term $\mathcal{L}_{\chi S B}$ explicitly breaks the symmetry.

In the vacuum one has $\langle\vec{\pi}\rangle=0$ by parity and one notes $\langle\sigma\rangle=f_{\pi}$ the constant expectation value of $\sigma$. The breaking of the symmetry by the vacuum $(\langle\sigma\rangle \neq 0)$ is realized at the classical level by imposing that the meson's energy be stationary at the point $\left(\sigma=f_{\pi}, \vec{\pi}=0\right)$. This amounts to 


$$
\frac{\partial}{\partial \sigma}\left[V_{\mathrm{pot}}\left(\sigma^{2}+\pi^{2}\right)-c \sigma\right]_{\sigma=f_{\pi}, \vec{\pi}=0}=0
$$

since the stationarity with respect to $\vec{\pi}$ is trivially satisfied. The other parameters are fixed by identifying the mass terms, that is,

$$
\begin{gathered}
g_{0}\langle\sigma\rangle=M_{N}, \quad m_{\sigma}^{2}=\left.\frac{\partial^{2} V_{\mathrm{pot}}}{\partial \sigma^{2}}\right|_{\sigma=f_{\pi}, \vec{\pi}=0}, \\
m_{\pi}^{2} \delta(i, j)=\left.\frac{\partial^{2} V_{\mathrm{pot}}}{\partial \pi_{i} \partial \pi_{j}}\right|_{\sigma=f_{\pi}, \vec{\pi}=0} .
\end{gathered}
$$

One gets

$$
c=m_{\pi}^{2} f_{\pi}, \quad \lambda=\frac{m_{\sigma}^{2}-m_{\pi}^{2}}{2 f_{\pi}^{2}}, \quad v^{2}=f_{\pi}^{2} \frac{m_{\sigma}^{2}-3 m_{\pi}^{2}}{m_{\sigma}^{2}-m_{\pi}^{2}},
$$

and the quantized version of the model is obtained by considering $\vec{\pi}$ and $\sigma^{\prime}=\sigma-f_{\pi}$ as the degrees of freedom.

This model is referred to as the linear sigma model (LSM). Since, in the limit $m_{\pi} \rightarrow 0$, its equations of motion respect chiral symmetry this model reproduces the soft pion theorems in the tree approximation. However, this generally involves somewhat unnatural cancellations between several diagrams. Moreover the lack of experimental evidence (see, however, Ref. [13]) for a scalar meson that could be associated with the fluctuation $\sigma^{\prime}$ has led to the idea that this field was unphysical and should be eliminated from the model. This is achieved by letting $m_{\sigma} \rightarrow \infty$ which leads to the constraint

$$
\sigma^{2}+\pi^{2}=f_{\pi}^{2}
$$

for the finite energy solutions. The constraint (15) is solved by the point transformation

$$
\sigma=f_{\pi} \cos F\left(\frac{\phi}{f_{\pi}}\right), \quad \vec{\pi}=\hat{\phi} \sin F\left(\frac{\phi}{f_{\pi}}\right),
$$

which eliminates the $\sigma$ field and defines $\vec{\phi}=\phi \hat{\phi}$ as a new pion field. $F$ is an odd function of the form

$$
F(x)=x+\alpha x^{3}+\cdots,
$$

which selects the particular realization of the model. Changing $F$ amounts to a redefinition of the pion field and thus should not affect the physics. In the following we keep $\alpha$ arbitrary and check that the final results do not depend on it.

The last step is to perform a new point transformation defined by [14]

$$
\psi=\exp \left(-\frac{1}{2} i \vec{\tau} \cdot \hat{\phi} F\left(\phi / f_{\pi}\right) \gamma_{5}\right) N
$$

and to take $N$ as the nucleon field. This defines the nonlinear sigma model. Due to the transformation (18), the pion then couples to the nucleon $N$ only through derivatives. This eliminates the unnatural cancellations of the LSM because the Born terms are automatically suppressed by powers of $m_{\pi}$ in the soft pion limit.

This is all fine for chiral symmetry but somewhat frustrating for nuclear physics. The reason is that the medium range attraction is known to be dominated by a scalar-isoscalar correlated two pion exchange. Chiral perturbation theory actually forbids the identification of this attraction with the exchange of the $\sigma^{\prime} \quad\left(=\sigma-f_{\pi}\right)$ field but, if we go back in the above discussion, we realize that the chiral radius $\sqrt{\sigma^{2}+\pi^{2}}$ has been fixed to $f_{\pi}$ by mere convenience. Nothing prevents us from keeping it as a degree of freedom and to, tentatively, identify it with the meson which produces the medium range attraction. To avoid any confusion with $\sigma$, the chiral partner of the pion, we shall note it as $\Theta$. The fact that no such meson is clearly seen in $\pi \pi$ scattering is not an obstacle. There is in the model a strong $\Theta \rightarrow \pi \pi$ coupling which, as in the linear sigma model, leads to a large $\Theta$ width. This may explain why this meson is so elusive. For the $N N$ interaction this large on-shell width of the $\Theta$ is not a conceptual difficulty because it comes into play only through spacelike exchange between nucleons. So its width is effectively zero.

We stress that, with respect to the LSM, we simply make a convenient change of variables $(\sigma, \vec{\pi}) \rightarrow(\Theta, \vec{\phi})$ which avoids keeping track of the cancellations inherent to the model. When studying elementary processes these cancellations are just a matter of care, but when they are intertwined with the unavoidable approximations of the nuclear manybody problem this may lead to results inconsistent with chiral symmetry.

\section{ALTERNATIVE FORMULATION OF THE LINEAR SIGMA MODEL}

Our starting point is defined by the Lagrange density

$$
\mathcal{L}=\mathcal{L}_{0}+\Delta \mathcal{L}_{0}+\mathcal{L}_{\chi S B},
$$

where to the symmetric piece $\mathcal{L}_{0}$ defined in Eq. (10) we have added, as in Ref. [4], the chiral invariant piece

$$
\Delta \mathcal{L}_{0}=i a \bar{\psi} \gamma^{\mu}\left(W \partial_{\mu} W^{\dagger} P^{L}+W^{\dagger} \partial_{\mu} W P^{R}\right) \psi,
$$

which is not present in the original sigma model. Its only role is to generate an axial coupling constant $g_{A}$ different from unity in the tree approximation. The spirit of this is not to try to make a realistic description of the nucleon but to make easier the identification of the evolution of this quantity. The axial current corresponding to Eq. (19) is

$$
\begin{aligned}
\vec{J}_{5 \mu}= & \left(1-\frac{a}{2} \operatorname{Tr} W^{\dagger} W\right)\left(\bar{\psi} \gamma_{\mu} \gamma_{5} \frac{\vec{\tau}}{2} \psi\right) \\
& -a \bar{\psi} \gamma_{\mu}\left(W^{\dagger} \frac{\vec{\tau}}{2} W P_{R}-W \frac{\vec{\tau}}{2} W^{\dagger} P_{L}\right) \psi \\
& -\frac{i}{4} \operatorname{Tr}\left(W^{\dagger} \partial_{\mu} W+W \partial_{\mu} W^{\dagger}\right),
\end{aligned}
$$


from which one sees that one needs

$$
a=\frac{1-g_{A}}{2 f_{\pi}^{2}}
$$

in order to get the correct value of the nucleon axial charge in the tree approximation. The other parameters $\left(\lambda, v, g_{0}, c\right)$ have the same expressions as in Eqs. (13),(14). Notice that if we note $Q_{i}^{5}$ the axial charge of the model, the symmetry breaking part of $\mathcal{L}$ is such that the identity $H_{\chi S B}$ $=\left[Q_{i}^{5},\left[Q_{i}^{5}, H\right]\right]$ is satisfied, as in QCD itself.

Guided by the discussion of Sec. II we make the point transformation $(\sigma, \vec{\pi}) \rightarrow(\Theta, \vec{\phi})$ defined by

$$
\sigma=\Theta \cos F\left(\frac{\phi}{f_{\pi}}\right), \quad \vec{\pi}=\Theta \hat{\phi} \sin F\left(\frac{\phi}{f_{\pi}}\right),
$$

which allows one to write

$$
W=\Theta U, \quad U=\xi^{2}=\exp \left[i \vec{\tau} \cdot \hat{\phi} F\left(\frac{\phi}{f_{\pi}}\right)\right]
$$

and we define the new nucleon field

$$
N=\left(\xi P^{R}+\xi^{\dagger} P^{L}\right) \psi,
$$

which is equivalent to Eq. (18). Note that the mass term $\bar{N} N$ is a chiral invariant. In the vacuum one has $\langle\Theta\rangle=\langle\sigma\rangle$ $=f_{\pi}$. So we define the fluctuation $\theta=\Theta-f_{\pi}$ and write $\mathcal{L}$ in terms of the degrees of freedom $(N, \theta, \vec{\phi})$, that is,

$$
\begin{aligned}
\mathcal{L}= & \left(f_{\pi}+\theta\right)^{2} \operatorname{Tr} \partial^{\mu} U \partial_{\mu} U^{\dagger}+\frac{1}{2} \partial^{\mu} \theta \partial_{\mu} \theta \\
& -\frac{m_{\sigma}^{2}-m_{\pi}^{2}}{8 f_{\pi}^{2}}\left(\theta^{2}+2 f_{\pi} \theta+\frac{2 f_{\pi}^{2} m_{\pi}^{2}}{m_{\sigma}^{2}-m_{\pi}^{2}}\right)^{2} \\
& +i \bar{N} \gamma^{\mu} \partial_{\mu} N-M_{N}\left(1+\frac{\theta}{f_{\pi}}\right) \bar{N} N+\bar{N} \gamma_{\mu} \mathcal{V}_{c}^{\mu} N \\
& +\left[1-\left(1-g_{A}\right)\left(1+\frac{\theta}{f_{\pi}}\right)^{2}\right] \bar{N} \gamma_{\mu} \gamma^{5} \mathcal{A}_{c}^{\mu} N \\
& +i \frac{1-g_{A}}{2 f_{\pi}}\left(1+\frac{\theta}{f_{\pi}}\right) \bar{N} \gamma^{\mu} N \partial_{\mu} \theta+\mathcal{L}_{\chi S B},
\end{aligned}
$$

where we have defined

$$
\mathcal{V}_{c}^{\mu}=\frac{i}{2}\left(\xi \partial_{\mu} \xi^{\dagger}+\xi^{\dagger} \partial_{\mu} \xi\right), \quad \mathcal{A}_{c}^{\mu}=\frac{i}{2}\left(\xi \partial_{\mu} \xi^{\dagger}-\xi^{\dagger} \partial_{\mu} \xi\right)
$$

We have

$$
m_{\theta}^{2}=\left.\frac{\partial^{2} \mathcal{L}(\theta)}{\partial \theta^{2}}\right|_{\theta=0}=m_{\sigma}^{2},
$$

so in the following $m_{\sigma}$ will be replaced by $m_{\theta}$.
In terms of the new variables, we get the following expressions for the symmetry breaking piece:

$$
\mathcal{L}_{\chi S B}=f_{\pi} m_{\pi}^{2}\left(f_{\pi}+\theta\right) \cos F\left(\frac{\phi}{f_{\pi}}\right),
$$

and for the axial current

$$
\begin{aligned}
\vec{J}_{5 \mu}= & -i \frac{f_{\pi}^{2}}{4}\left(1+\theta / f_{\pi}\right)^{2} \operatorname{Tr}\left(\vec{\tau} U^{\dagger} \partial_{\mu} U-\vec{\tau} U \partial_{\mu} U^{\dagger}\right) \\
& +\frac{1}{2}\left[1-\left(1-g_{A}\right)\left(1+\theta / f_{\pi}\right)^{2}\right] \bar{N} \gamma_{\mu} \gamma^{5} \\
& \times\left(\xi \frac{\tau_{i}}{2} \xi^{\dagger}+\xi^{\dagger} \frac{\tau_{i}}{2} \xi\right) N+\frac{1}{2} \bar{N} \gamma_{\mu}\left(\xi \frac{\tau_{i}}{2} \xi^{\dagger}-\xi^{\dagger} \frac{\tau_{i}}{2} \xi\right) N .
\end{aligned}
$$

Some comments on the Lagrangian of Eq. (26) are in order. The term $\bar{N} \gamma_{\mu} \gamma^{5} \mathcal{A}_{c}^{\mu} N$ generates the standard $\pi N N \quad p$-wave coupling but corrected by a $3 \pi N N$ coupling and other higher order terms. One can check that the Goldberger relation $g_{\pi N N} f_{\pi}=M_{N} g_{A}$ is fulfilled. There is a nonderivative $\theta N$ interaction with a coupling constant equal to $M_{N} / f_{\pi} \propto 10$ which is smaller than the $\pi N$ coupling constant by a factor $1 / g_{A}$. Note that this coupling constant is not a free parameter in this model because all the nucleon mass is generated by the spontaneous symmetry breaking. This will no longer be true in models where part of the nucleon mass is due to the confinement. Finally we stress that, in the chiral limit, the new scalar field $\theta$ couples only derivatively to two pions, there is no term of the form $\theta \phi^{2}$. This insures the validity of the soft pion theorems for $\pi N$ scattering.

\section{MEDIUM EFFECTS}

We are now in a situation to describe various in-medium quantities in the framework of the mean field approximation combined with the pion gas limit.

\section{A. Condensate evolution}

Firstly the quark condensate and its evolution at finite density can be obtained by identifying the symmetry breaking pieces of QCD and the one of our Lagrangian, that is,

$$
-2 m_{q} \bar{q} q \leftrightarrow f_{\pi} m_{\pi}^{2} \sigma=f_{\pi} m_{\pi}^{2}\left(f_{\pi}+\theta\right) \cos F\left(\frac{\phi}{f_{\pi}}\right) .
$$

This equation shows that the condensate evolution is driven by the mean value of $\sigma$, the chiral partner of the pion. From Eq. (31) and using the Gell-Mann, Oakes, and Renner relation, we get the relative modification of the condensate

$$
\frac{\langle\bar{q} q(\rho)\rangle}{\langle\bar{q} q(0)\rangle}=1-\frac{\left\langle\phi^{2}\right\rangle}{2 f_{\pi}^{2}}+\left\langle\frac{\theta}{f_{\pi}}\left(1-\frac{\phi^{2}}{2 f_{\pi}^{2}}\right)\right\rangle+\cdots,
$$

where we have expanded $\cos F\left(\phi / f_{\pi}\right)$ and kept only the leading terms in $1 / f_{\pi}$. There are two contributions to the resto- 


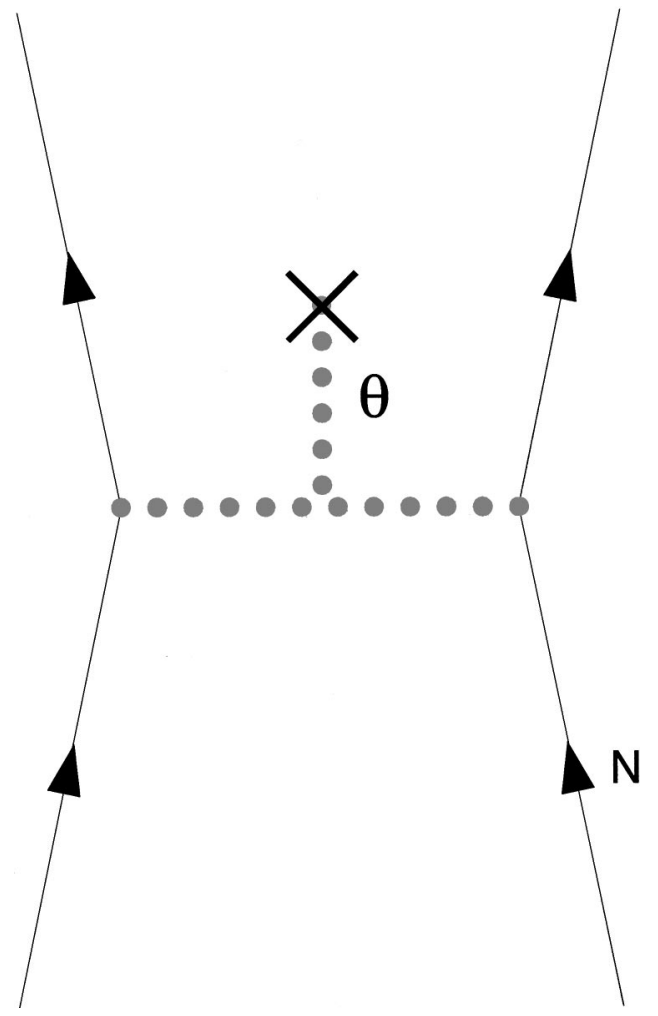

FIG. 1. The mechanism which induces the term quadratic in $\rho$ in Eq. (34).

ration effect. The first one arises from the pion cloud, the second one is driven by the scalar field $\theta$. The second contribution also depends on the squared pion field, which is to be expected since the condensate is not a chiral invariant quantity.

The mean field $\langle\theta\rangle$ is obtained from the equation of motion which writes, for a uniform medium of density $\rho$ :

$$
\frac{f_{\pi} m_{\theta}^{2}}{2}\left(2 X+3 X^{2}+X^{3}\right)+g_{0} \rho=0, \quad \text { with } \quad X=\frac{\langle\theta\rangle}{f_{\pi}},
$$

where terms of order $m_{\pi}^{2}$ have been ignored. To second order in the density the solution is

$$
\frac{\langle\theta\rangle}{f_{\pi}}=-\frac{g_{0} \rho}{f_{\pi} m_{\theta}^{2}}-\frac{3}{2}\left(\frac{g_{0} \rho}{f_{\pi} m_{\theta}^{2}}\right)^{2} .
$$

To zeroth order in the pion field the quantity $\langle\theta\rangle / f_{\pi}$ fixes the relative amount of restoration from the $\theta$ exchange. Numerical estimates will be discussed later.

The term quadratic in the density in Eq. (34) represents a moderate effect at normal density but its interpretation is interesting. Due to the $\theta^{3}$ vertex in Eq. (26), the mean field $\langle\theta\rangle$ gets a contribution from the $\theta$ exchange as shown in Fig. 1. For what concerns the condensate evolution this second order term in $\rho$ represents the contribution to the restoration due to the $\theta$ exchanged between nucleons. Indeed the sigma commutator of the $\theta$ meson $\Sigma_{\theta}$ which fixes the amount of restoration induced by a single $\theta$, can be obtained from the Feynman-Hellman theorem, which leads to

$$
\Sigma_{\theta}=\left.c \frac{d m_{\theta}}{d c}\right|_{\lambda, v}=\left.3 c \frac{m_{\pi}}{m_{\theta}} \frac{d m_{\pi}}{d c}\right|_{\lambda, v}=3 \frac{m_{\pi}}{m_{\theta}} \Sigma_{\pi}=\frac{3 m_{\pi}^{2}}{2 m_{\theta}} .
$$

This quantity has to be multiplied by the two-body contribution to the $\theta$ scalar density $\rho_{\theta}^{(2)}$ which is

$$
\rho_{\theta}^{(2)}=m_{\theta} \int d \vec{x}_{1} d \vec{x}_{2} \rho\left(\vec{x}_{1}\right) \rho\left(\vec{x}_{2}\right) \theta_{1}(\vec{x}) \theta_{2}(\vec{x})=\frac{g_{0}^{2} \rho^{2}}{m_{\theta}^{3}}
$$

where $\theta_{i}$ is the $\theta$ field created by the nucleon located at $\vec{x}_{i}$. In order to obtain the relative amount of restoration we have to multiply Eq. (35) by expression (36) and divide by $\left(-f_{\pi}^{2} m_{\pi}^{2}\right)$. This gives $3\left(g_{0} \rho / m \sigma^{2}\right)^{2} / 2$, which is precisely the quadratic part of Eq. (34). This second order term in the density of the quark condensate was already given by Birse and McGovern [10]. It is absent in the nonlinear sigma model [11], as it should be since this correction concerns only the $\theta$ meson. Weise [15] also estimated the contribution to the restoration of the sigma exchanged between nucleons by relating the sigma commutator of the sigma to the nucleon one.

\section{B. Nucleon and $\theta$ mass evolution}

We now come to another in-medium quantity, the effective nucleon mass. Its evolution in presence of the mean scalar field is apparent from the Lagrangian (19). It reads

$$
M_{N}^{*}=M_{N}-g_{0}\langle\theta\rangle=M_{N}-\frac{g_{0}^{2} \rho}{m_{\theta}^{2}}-\frac{3 g_{0}}{2 f_{\pi}}\left(\frac{g_{0}^{2} \rho}{m_{\theta}^{2}}\right)^{2} .
$$

It is exclusively governed by the chiral invariant scalar field $\theta$. It has no dependence at all on $\phi^{2}$, which eliminates the conflict with the chiral perturbation constraints. The cancellations of the linear model are indeed present for the mass, in such a way that the influence of the pion loops on the mass is eliminated. The present formulation of the model automatically insures these cancellations. The identification with the mean scalar field of the Walecka model now becomes obvious. The scalar field of QHD has to be identified with the scalar invariant mean field $\langle\theta\rangle$ and not with $\sigma^{\prime}=\sigma-f_{\pi}$, the chiral partner of the pion. The model also provides its coupling to the nucleon, $g_{\theta N N}=g_{0}=M / f_{\pi} \simeq 10$, somewhat smaller than $g_{\pi n N}=13.5$, a welcome feature with respect to the phenomenology of QHD [5].

The chiral invariant character of the mean scalar field of QHD is not a new concept. It was first studied by Lin and Serot [16] in the framework of the linear sigma model combined with dispersion relations to account for the exchange of correlated pions in the $N N$ interaction. In the work of Serot [17] a chiral invariant scalar is added to the nonlinear sigma model but its coupling to the nucleon is arbitrary and the in-medium mass bears no relation to the condensate evo- 
lution. On the other hand Delorme et al. [18] have studied the nucleon mass in the quark-meson coupling model [19]. Here the source of the scalar field is the quark scalar density, which allows a link between the nucleon mass and the condensate modifications. The chiral invariant character of the quark-meson coupling was imposed.

We are now in a situation to discuss the relation between the mass and condensate evolutions, a connection totally absent in the standard formulation of QHD, and the connection between the present work and the scaling law of Brown and Rho. The mass evolution is related to the condensate evolution, but only to part of it, the purely nonpionic part. Only the chiral invariant field $\theta$ influences the mass. This $\theta$ field is dressed by the pion loops while the $\sigma$ is not. It is only to zero order in the pion loops that the two evolutions are the same. Note that, to zeroth order in the pion loops and in the low density limit, our approach gives $\alpha=1$ instead of $\alpha=1 / 3$ for the expression of the scaling factor of Eq. (6).

In the same way the effective $\theta$ mass follows from the Lagrangian (26). In the nuclear medium the $\theta$ field acquires mean value $\langle\theta\rangle$ and the effective mass refers to the fluctuations about this mean value, that is,

$$
m_{\theta}^{* 2}=\left.\frac{\partial^{2} \mathcal{L}(\theta)}{\partial \theta^{2}}\right|_{\theta=\langle\theta\rangle},
$$

which leads to

$$
\left(\frac{m_{\theta}^{*}}{m_{\theta}}\right)^{2}=1+3 \frac{\langle\theta\rangle}{f_{\pi}}+\frac{3\langle\theta\rangle^{2}}{2 f_{\pi}^{2}}=1-\frac{3 g_{0} \rho}{f_{\pi} m_{\theta}^{2}} .
$$

It turns out that in Eq. (39) there is a cancellation between the terms which are quadratic in $\rho$. The $\theta$ mass is reduced by the medium effects getting closer to the pion mass. It follows a pattern similar to that of the nucleon mass, with a somewhat faster evolution, as seen by comparing Eqs. (37), (39). Thus in the nuclear medium, the shape of the Mexican hat $\left(V_{\text {pot }}\right)$ is appreciably modified. There is not only a shrinking of the radius of the chiral valley due to the mean value of the $\theta$ field, but accordingly the potential becomes more shallow. The lowering of the $\theta$ mass suggests enhanced fluctuations around the mean value. The connection between chiral symmetry restoration and the sigma mass as well as the experimental implications have been studied by Hatsuda et al. [20].

We now make some numerical evaluations. We have five paramaters in our version of the model. They are linked, through the set of equations (13),(14), to the pion decay constant, the nucleon mass, the axial coupling constant and the pion and the theta masses. All these quantities are measured, except for the $\theta$ mass which can be taken as a free parameter. As an example we will take two values $m_{\theta}=1 \mathrm{GeV}$ and $m_{\theta}=0.8 \mathrm{GeV}$. For $m_{\theta}=1 \mathrm{GeV}(0.8 \mathrm{GeV})$ the scalar mean field $\theta$ has a value of $17 \mathrm{MeV},(30 \mathrm{MeV})$ at normal density. The corresponding nucleon mass reduction is $M_{N}^{*}-M_{N}$ $=-170 \mathrm{MeV}(-300 \mathrm{MeV})$. These magnitudes are compatible with the current phenomenology of QHD. According to Eq. (39), the $\theta$ mass also drops by $22 \%$ (34\%), an appreciable modification. For the condensate evolution we remind the reader that the pion cloud, that is the term $\left\langle\phi^{2}\right\rangle / 2 f_{\pi}^{2}$ in Eq. (32), produces a relative decrease of about $20 \%$. The part of the condensate evolution due to the scalar field depends not only on the expectation value $\left\langle\theta / f_{\pi}\right\rangle$ but also on $\left\langle\left(\theta / f_{\pi}\right)\left(\phi^{2} / 2 f_{\pi}^{2}\right)\right\rangle$ which we estimate as $\left\langle\theta / f_{\pi}\right\rangle\left\langle\phi^{2} / 2 f_{\pi}^{2}\right\rangle$. In this way we find a relative decrease of $14 \%(25 \%)$ of the condensate.

\section{Evolution of $g_{A}$}

To order $\phi^{3}$ the axial current (30) is written

$$
\begin{aligned}
\vec{J}^{\mu 5}= & f_{\pi}\left(1+\frac{2 \theta}{f_{\pi}}\right)\left[\left(1+3 \alpha \frac{\phi^{2}}{f_{\pi}^{2}}\right) \partial^{\mu} \vec{\phi}+\frac{1}{f_{\pi}^{2}}\left(2 \alpha+\frac{2}{3}\right)\right. \\
& \left.\times\left(\vec{\phi} \vec{\phi} \cdot \partial^{\mu} \vec{\phi}-\phi^{2} \partial^{\mu} \vec{\phi}\right)\right]+g_{A}\left(1+2 \frac{g_{A}-1}{g_{A}} \frac{\theta}{f_{\pi}}\right) \\
& \times\left[\bar{N} \gamma^{\mu} \gamma^{5} \frac{\vec{\tau}}{2} N+\frac{1}{2 f_{\pi}^{2}} \bar{N} \gamma^{\mu} \gamma^{5}\left(\vec{\phi} \frac{\vec{\phi} \cdot \vec{\tau}}{2}-\phi^{2} \frac{\vec{\tau}}{2}\right) N\right] \\
& +\frac{1}{f_{\pi}} \bar{N} \gamma^{\mu} \frac{\vec{\phi} \times \vec{\tau}}{2} N+\cdots .
\end{aligned}
$$

The last term in Eq. (40) does not contribute in the mean field approximation. The medium modification is due to the coupling to the $\theta$ field and to the terms with several pion fields. In the mean field approximation we replace $\theta$ by $\langle\theta\rangle$ and $\phi^{i} \phi^{j}$ by $\left\langle\phi^{i} \phi^{j}\right\rangle=\left\langle\phi^{2}\right\rangle \delta_{i j} / 3$ which gives the mean current

$$
\begin{aligned}
\left\langle\vec{J}^{\mu 5}\right\rangle= & f_{\pi}\left(1+\frac{2\langle\theta\rangle}{f_{\pi}}\right)\left[1+\frac{\left\langle\phi^{2}\right\rangle}{f_{\pi}^{2}}\left(\frac{5 \alpha}{3}-\frac{4}{9}\right)\right] \partial^{\mu} \vec{\phi} \\
& +g_{A}\left(1+2 \frac{g_{A}-1}{g_{A}} \frac{\langle\theta\rangle}{f_{\pi}}\right)\left(1-\frac{\left\langle\phi^{2}\right\rangle}{3 f_{\pi}^{2}}\right) \bar{N} \gamma^{\mu} \gamma^{5} \frac{\vec{\tau}}{2} N,
\end{aligned}
$$

from which, to lowest order in $\langle\theta\rangle$ and $\left\langle\phi^{2}\right\rangle$, we get the following expression for the evolution of $g_{A}$ :

$$
\frac{g_{A}^{*}}{g_{A}}=\left(1+2 \frac{g_{A}-1}{g_{A}} \frac{\langle\theta\rangle}{f_{\pi}}-\frac{2}{3} \frac{\left\langle\phi^{2}\right\rangle}{2 f_{\pi}^{2}}\right) .
$$

At normal nuclear density the scalar contribution yields a quenching of $g_{A}$ of the order of $6 \%$ while the pionic contribution gives a quenching of about $15 \%$. As pointed out in Ref. [4], this result is strictly valid only when the short range correlations between nucleons are neglected. The renormalizations of the weak coupling constants $g_{A}$ and $f_{\pi}$, due to the suppression of the quark condensate by the scalar meson have been previously discussed by Akmedov [21] in the usual formulation of the sigma model, ignoring the pion loops. 


\section{Pionic properties evolution}

We now turn to the in-medium values of pionic properties: the pion decay constant and the pion mass. We stress that we are concerned only by the influence of the two mesons present in our model $\theta$ and $\pi$. In this context the nucleons act only as a source for these fields. The problem reduces to the question of the pion mass and decay constant in a pion gas and in the presence of a mean scalar field $\langle\theta\rangle$. Within this limited framework we do not expect a realistic description of the in-medium effects for these two quantities. Indeed the pion mass modification is linked, in the dilute gas limit, to the isospin symmetric $\pi N$ amplitude and is of order $m_{\pi}^{2}$. It is subject to other influences than just the pion and the theta. The pion decay constant which is linked to the pion mass by the Gell-Mann, Oakes, and Renner relation is also subject to these extra influences. Therefore we quote the implications of the model for $f_{\pi}$ and $m_{\pi}$ only to show the absence of a universal link between their evolution and the condensate one.

For what concerns the influence of the nuclear pion gas, it has already been studied [4,22]. It is described through the scalar pion density (5). On this particular point the present work brings nothing new. The novel part concerns the influence of the $\theta$ field. For completeness, however, we treat the two effects simultaneously in our formulation of the linear sigma model.

The effective pion decay constant is the coefficient of $\partial^{\mu} \vec{\phi}$ in the mean axial current (41) multiplied by the wave function renormalization $\sqrt{Z}$ (see the Appendix). To leading order in $\langle\theta\rangle$ and $\left\langle\phi^{2}\right\rangle$ we get the result, independent of $\alpha$ as it should be

$$
\frac{f_{\pi}^{*}}{f_{\pi}}=\left(1+\frac{\langle\theta\rangle}{f_{\pi}}-\frac{2}{3} \frac{\left\langle\phi^{2}\right\rangle}{2 f_{\pi}^{2}}\right) .
$$

We see that the evolution of $f_{\pi}$ follows the condensate, Eq. (32), only for what concerns the scalar field piece. At variance with the nucleon mass case there is a pionic piece. With respect to the condensate evolution (32) this pionic term is multiplied by $2 / 3$, exactly as in the thermal case.

The effective pion mass is defined as the position of the pole energy of the propagator for vanishing threemomentum. It obeys the relation

$$
m_{\pi}^{* 2}=m_{\pi}^{2}+S\left(m_{\pi}^{*}, \overrightarrow{0}\right)
$$

where $S(q)$ is the pion self-energy. As shown in the Appendix this leads to

$$
\left(\frac{m_{\pi}^{*}}{m_{\pi}}\right)^{2}=\left(1-\frac{\langle\theta\rangle}{f_{\pi}}+\frac{\left\langle\phi^{2}\right\rangle}{6 f_{\pi}^{2}}\right)
$$

Both terms on the RHS of Eq. (44) are positive, corresponding to a repulsive interaction and it is clear that the evolution of $m_{\pi}$ is completly different from the condensate one.

\section{CONCLUSION}

In this work we have studied the role of the scalar meson both in the partial restoration of chiral symmetry and in the lowering of the hadron mass in the nuclear medium, as well as the link between the two effects in the framework of the linear sigma model. We have used a formulation of the linear sigma model with the usual nonlinear realization for the pion field but we have kept a scalar degree of freedom corresponding to the fluctuation along the chiral radius. This new scalar field is not the chiral partner of the pion but instead is a chiral invariant. It is already dressed by the pion loops. Its mean value in the medium represents the modification of the radius of the chiral circle as compared to the vacuum value. In this formalism the low-energy theorems and the constraints of chiral perturbation theory are easily fulfilled without need for cancellations. For instance, this scalar $\theta$ couples derivatively to two pions, in the chiral limit. For what concerns the density evolution of the quark condensate, which is not a chiral invariant quantity, it is instead governed by the mean sigma field, the chiral partner of the pion. This difference shows up in the comparison between the two evolutions. The condensate is influenced by the pion cloud while the mass is not. It is only in zero order in the pion loops that the two relative evolutions become the same. In practice the difference is large since about half of the restoration originates from the pion cloud.

Our work shows that for what concerns the scalar field, quantum hadrodynamics can be incorporated in a chiral theory such as the linear sigma model. The scalar field of QHD should be identified with the chiral invariant scalar field $\theta$. This is in fact imposed by the constraints of chiral perturbation theory, which prevents the nucleon-nucleon potential from being influenced by the pion density in the chiral limit. The phenomenology which comes out from this reformulation is compatible with that of QHD.

Concerning the signals associated with the restoration they are of two types depending on the origin of the restoration. In the linear sigma model the quark condensate depends on the average sigma field. In the vacuum $\langle\sigma\rangle=f_{\pi}$. In the medium this quantity is modified by two effects. On the one hand, there is an oscillation along the chiral circle induced by the pionic fluctuations, which is described by pion density. On the other hand, there is a modification of the radius of the chiral circle due to the mean scalar field $\theta$. The oscillation along the chiral circle shows up in the mixing of the axial and vector correlators. The shrinking of the chiral radius shows up in the lowering of the nucleon mass. Both types of signal are simultaneously present in the nuclear medium.

\section{ACKNOWLEDGMENTS}

We have benefited from discussions with M. Birse. This work was initiated during the stay of two of us (M.E. and P.A.M.G.) at the SRCSSM of Adelaide. We thank A.W. Thomas and A. Williams for their support and the stimulating atmosphere of the SRCSSM. 


\section{APPENDIX}

We study the pion propagation in the nuclear medium. In addition to the excitation of particle-hole states by the $s$-and $p$-wave couplings, the in-medium pion self-energy $S$ receives a contribution from the pion loop. For a pion of fourmomentum $q$ and isospin label $a$ the one loop self-energy is written

$$
\begin{aligned}
S^{\text {loop }}(q) & \equiv S^{\text {loop }}(\omega \vec{q}) \\
& =\frac{1}{2} \int \frac{i d^{4} k}{(2 \pi)^{4}} \sum_{c}\langle q a ; k c|\mathcal{M}| q a ; k c\rangle D_{R}(k),
\end{aligned}
$$

where $D_{R}(k)$ is the full in-medium pion propagator and $\mathcal{M}$ is the (possibly in medium modified) $\pi \pi$ interaction which has the decomposition

$$
\begin{aligned}
\left\langle q_{1} a ;\right. & \left.k_{1} b\left|\mathcal{M}_{0}\right| q_{2} c ; k_{2}, d\right\rangle \\
= & \left\langle q_{1} ; k_{1}\left|\mathcal{M}_{s}\right| q_{2} ; k_{2}\right\rangle \delta_{a b} \delta_{c d}+\left\langle q_{1} ; k_{1}\left|\mathcal{M}_{t}\right| q_{2} ; k_{2}\right\rangle \\
& \times \delta_{a c} \delta_{b d}+\left\langle q_{1} ; k_{1}\left|\mathcal{M}_{u}\right| q_{2} ; k_{2}\right\rangle \delta_{a d} \delta_{b c},
\end{aligned}
$$

and the projection on the total isospin states $I=0,1,2$ of the $s$ channel $\left[s=(p+q)^{2}\right]$ are

$$
\begin{gathered}
\mathcal{M}_{0}=3 \mathcal{M}_{s}+\mathcal{M}_{t}+\mathcal{M}_{u}, \quad \mathcal{M}_{1}=\mathcal{M}_{t}-\mathcal{M}_{u}, \\
\mathcal{M}_{2}=\mathcal{M}_{t}+\mathcal{M}_{u} .
\end{gathered}
$$

Working out the isospin summations one finds

$$
\begin{aligned}
S^{\operatorname{loop}}(q)= & \frac{1}{2} \int \frac{i d^{4} k}{(2 \pi)^{4}} \sum_{c}\langle q ; k| \frac{1}{3} \\
& \times\left(\mathcal{M}_{0}+3 \mathcal{M}_{1}+5 \mathcal{M}_{2}\right)|q ; k\rangle D_{R}(k) .
\end{aligned}
$$

The particular combination $\frac{1}{3}\left(\mathcal{M}_{0}+3 \mathcal{M}_{1}+5 \mathcal{M}_{2}\right)=3 \mathcal{M}_{t}$ $+\mathcal{M}_{s}+\mathcal{M}_{u}$ is in fact the $I=0$ amplitude of the $t$ channel

$$
S^{\operatorname{loop}}(q)=\frac{1}{2} \int \frac{i d^{4} k}{(2 \pi)^{4}} \sum_{c}\left\langle q ;-q\left|\mathcal{M}_{0}\right| k ;-k\right\rangle D_{R}(k) .
$$

Let us calculate, in the tree approximation, this $I$ $=0, \quad \pi \pi$ amplitude, first ignoring possible in-medium vertex corrections. The relevant piece of the Lagrangian is

$$
\mathcal{L}_{\pi \pi}=\left(f_{\pi}+\theta\right)^{2} \frac{1}{4} \operatorname{Tr} \partial_{\mu} U \partial^{\mu} U^{\dagger}+f_{\pi} m_{\pi}^{2}\left(f_{\pi}+\theta\right) \cos F\left(\frac{\phi}{f_{\pi}}\right) .
$$

At the tree level we keep the terms of order $\phi^{4}$ and the $\theta \pi \pi$ interaction term

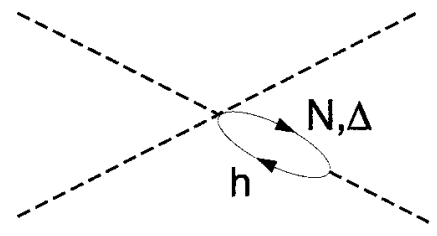

FIG. 2. Vertex correction to $\pi \pi$ scattering.

$$
\begin{aligned}
\mathcal{L}_{\pi \pi}^{(4)}= & \frac{1}{f_{\pi}^{2}}\left[-m_{\pi}^{2}\left(\alpha-\frac{1}{24}\right) \phi^{4}+\left(\alpha-\frac{1}{6}\right) \phi^{2} \partial_{\mu} \vec{\phi} \cdot \partial^{\mu} \vec{\phi}\right. \\
& \left.+\left(2 \alpha+\frac{1}{6}\right) \vec{\phi} \cdot \partial_{\mu} \vec{\phi} \vec{\phi} \cdot \partial^{\mu} \vec{\phi}\right], \\
& \mathcal{L}_{\pi \pi}^{\theta \pi \pi}=\frac{\theta}{f_{\pi}}\left(\partial_{\mu} \vec{\phi} \cdot \partial^{\mu} \vec{\phi}-\frac{1}{2} m_{\pi}^{2} \phi^{2}\right) .
\end{aligned}
$$

The $\mathcal{M}_{s}$ amplitude is straightforwardly obtained as

$$
\begin{aligned}
\left\langle q_{a} ; q_{b}\left|\mathcal{M}_{s}\right| q_{c} ; q_{d}\right\rangle & \\
= & \frac{1}{f_{\pi}^{2}}\left[-\left(s-m_{\pi}^{2}\right)-2(\alpha-1 / 6) \sum_{i=a, \ldots, d}\left(q_{i}^{2}-m_{\pi}^{2}\right)\right. \\
& \left.+\left(s-q_{a}^{2}-q_{b}^{2}+m_{\pi}^{2}\right)\left(s-q_{c}^{2}-q_{d}^{2}+m_{\pi}^{2}\right) / s-m_{\theta}^{2}\right],
\end{aligned}
$$

where $s=\left(q_{a}+q_{b}\right)^{2}=\left(q_{c}+q_{d}\right)^{2}$ is the squared c.m. energy of the pion pair. We see from Eq. (A9) that in the low-energy regime of interest $\left(q \sim m_{\pi}\right)$ the $\theta$ exchange contribution is of order $m_{\pi}^{2} / m_{\theta}^{2}$. Since we limit ourselves to the leading order in the chiral expansion we only keep the first contribution on the RHS of Eq. (A9), which is nothing but the well-known nonlinear sigma model result.

The $\mathcal{M}_{t}$ and $\mathcal{M}_{u}$ amplitudes are obtained by the substitution ( $a \leftrightarrow c, s \leftrightarrow t)$ and $(a \leftrightarrow d, s \leftrightarrow u)$, respectively. It follows that the $I=0$ amplitude reads

$$
\begin{aligned}
\left\langle q_{a} ; q_{b}\left|\mathcal{M}_{0}\right| q_{c} ; q_{d}\right\rangle & =\frac{1}{f_{\pi}^{2}}\left[m_{\pi}^{2}-2 s+\beta \sum_{i=a, \ldots, d}\left(m_{\pi}^{2}-q_{i}^{2}\right)\right], \\
\beta & =1+10(\alpha-1 / 6),
\end{aligned}
$$

where the relation $s+t+u=\sum_{i=a, \ldots, d} q_{i}^{2}$ has been used.

In the medium Chanfray and Davesne [22] have established that the $\pi \pi$ interaction receives vertex corrections of the type shown in Fig. 2. The $3 \pi N$ vertex derived from the Lagrangian (A20) is, at the relevant order

$$
\begin{aligned}
\mathcal{L}_{3 \pi N}= & \frac{g_{A}}{2 f_{\pi}^{3}} \bar{N} \gamma^{\mu} \gamma_{5} \vec{\tau} N\left[(\alpha-1 / 6) \phi^{2} \partial_{\mu} \vec{\phi}\right. \\
& \left.+(2 \alpha+1 / 6) \vec{\phi} \vec{\phi} \cdot \partial_{\mu} \vec{\phi}\right] .
\end{aligned}
$$

For a zero momentum pion pair $(\vec{P}=0)$ the effective inmedium $I=0, \pi \pi$ potential takes the simple form 


$$
\begin{aligned}
& \left\langle q_{a} ; q_{b}\left|\mathcal{M}_{0}^{\mathrm{eff}}\right| q_{c} ; q_{d}\right\rangle \\
& =\frac{1}{f_{\pi}^{2}}\left[m_{\pi}^{2}-2 s+\beta \sum_{i=a, \ldots, d}\left[m_{\pi}^{2}-q_{i}^{2}+\vec{q}_{i}^{2} \widetilde{\Pi}^{0}\left(\omega_{i}, \vec{q}_{i}\right)\right]\right],
\end{aligned}
$$

where $\vec{q}_{i}^{2} \widetilde{\Pi}^{0}\left(\omega_{i}, \vec{q}_{i}\right)$ is the standard $p$-wave pionic polarizability which may include the screening effect from short range correlations. Hence the effect of vertex corrections depending on the $p$-wave polarizabilities is to make the effective $\pi \pi$ potential independent of $\alpha$ for on shell quasipions satisfying

$$
m_{\pi}^{2}-q_{i}^{2}+\vec{q}_{i}^{2} \widetilde{\Pi}^{0}\left(\omega_{i}, \vec{q}_{i}\right)=0 .
$$

The pion loop contribution to the pion self-energy (A5) is obtained from the matrix element of the $I=0$ amplitude making the replacements $q_{a}=q, q_{b}=-q, q_{c}=k, q_{d}=-k, s$ $=t=0$ :

$$
\begin{aligned}
S^{\operatorname{loop}}(q)= & \frac{1}{2} \int \frac{i d^{4} k}{(2 \pi)^{4}}\left\{m_{\pi}^{2}-2 \beta\left[D_{R}^{-1}(k)+\omega^{2}-\vec{q}^{2}\right.\right. \\
& \left.\left.-m_{\pi}^{2}-\vec{q}^{2} \widetilde{\Pi}^{0}(\omega, \vec{q})\right]\right\} D_{R}(k) .
\end{aligned}
$$

Since we are interested in the effect of the in-medium pion cloud we have to subtract the vacuum contribution. Constant terms such as $D^{-1} D$ disappear. One obtains

$$
\begin{aligned}
S^{\operatorname{loop}}(q)= & \frac{1}{2} \int \frac{i d^{4} k}{(2 \pi)^{4}}\left\{m_{\pi}^{2}-2 \beta\left[\omega^{2}-\vec{q}^{2}-m_{\pi}^{2}\right.\right. \\
& \left.\left.-\vec{q}^{2} \widetilde{\Pi}^{0}(\omega, \vec{q})\right]\right\}\left[D_{R}(k)-D_{0}(k)\right] \\
= & \frac{\left\langle\phi^{2}\right\rangle}{6 f_{\pi}^{2}}\left\{m_{\pi}^{2}-2 \beta\left[\omega^{2}-\vec{q}^{2}-m_{\pi}^{2}-\vec{q}^{2} \widetilde{\Pi}^{0}(\omega, \vec{q})\right]\right\},
\end{aligned}
$$

which is valid to leading order in the pion density defined by

$$
\left\langle\phi^{2}\right\rangle=3 \int \frac{i d^{4} k}{(2 \pi)^{4}}\left[D_{R}(k)-D_{0}(k)\right]
$$

[1] E. G. Drukarev and E. M. Levin, Nucl. Phys. A511, 679 (1990).

[2] T. D. Cohen, R. J. Furnstahl, and D. K. Griegel, Phys. Rev. C 45, 1881 (1992).

[3] M. Dey, V. L. Eletsky, and B. L. Ioffe, Phys. Lett. B 252, 620 (1990).

[4] G. Chanfray, J. Delorme, and M. Ericson, Nucl. Phys. A637, 421 (1998).

[5] B. D. Serot and J. D. Walecka, Adv. Nucl. Phys. 16, 1 (1986).

[6] B. D. Serot and J. D. Walecka, Int. J. Mod. Phys. E 16, 515 (1997).

[7] G. E. Brown and M. Rho, Phys. Rev. Lett. 66, 2720 (1991).
Finally the pion self-energy has a contribution from the scalar field which can be obtained directly from the $\pi \pi \theta$ Lagrangian

$$
S^{(\theta)}(q)=-\left(2 q^{2}-m_{\pi}^{2}\right) \frac{\langle\theta\rangle}{f_{\pi}} .
$$

The pion self-energy is

$$
\begin{aligned}
S(q)= & S^{(p \text {-wave })}(q)+S^{(\text {loop })}(q)+S^{(\theta)}(q) \\
= & \vec{q}^{2} \widetilde{\Pi}^{0}(\omega, \vec{q})+\frac{\left\langle\phi^{2}\right\rangle}{6 f_{\pi}^{2}}\left\{m_{\pi}^{2}-2 \beta\left[\omega^{2}-\vec{q}^{2}-m_{\pi}^{2}\right.\right. \\
& \left.\left.-\vec{q}^{2} \widetilde{\Pi}^{0}(\omega, \vec{q})\right]\right\}-\left(2 q^{2}-m_{\pi}^{2}\right) \frac{\langle\theta\rangle}{f_{\pi}} .
\end{aligned}
$$

In the above expression we have ignored the $s$ wave coupling. It would influence the pion mass though the Born part of the $\pi N$ amplitude. The pion propagator at $\vec{q}=0$ is written

$$
\widetilde{D}_{R}(\omega)=\left[\omega^{2}-m_{\pi}^{2}-S(q)\right]^{-1}=\frac{Z}{\omega^{2}-m_{\pi}^{* 2}},
$$

with

$$
Z=1-\frac{\beta\left\langle\phi^{2}\right\rangle}{3 f_{\pi}^{2}}-\frac{2\langle\theta\rangle}{f_{\pi}^{2}}
$$

from which we deduce the effective pion mass, to lowest order in $\left\langle\phi^{2}\right\rangle$ and $\langle\theta\rangle$

$$
\left(\frac{m_{\pi}^{*}}{m_{\pi}}\right)^{2}=Z\left(1+(1+2 \beta) \frac{\left\langle\phi^{2}\right\rangle}{6 f_{\pi}^{2}}+\frac{\langle\theta\rangle}{f_{\pi}}\right)=\left(1-\frac{\langle\theta\rangle}{f_{\pi}}+\frac{\left\langle\phi^{2}\right\rangle}{6 f_{\pi}^{2}}\right)
$$

As it should be the result is independent of $\alpha$, that is independent of the choice of the canonical pion field.
[8] M. Birse, Phys. Rev. C 53, 2048 (1996).

[9] M. Birse, Acta Phys. Pol. B 29, 2357 (1998).

[10] M. C. Birse and S. S. McGovern, Phys. Lett. B 309, 231 (1993).

[11] J. Delorme, G. Chanfray, and M. Ericson, Nucl. Phys. A603, 239 (1996)

[12] M. Gell-Mann and M. Levy, Nuovo Cimento 16, 705 (1958).

[13] N. A. Tornqvist and M. Roos, Phys. Rev. Lett. 76, 1575 (1996).

[14] S. Weinberg, Phys. Rev. 166, 1568 (1968).

[15] W. Weise, Nucl. Phys. A610, 35c (1996).

[16] W. Lin and B. Serot, Phys. Lett. B 233, 23 (1989). 
[17] R. J. Furnstahl, B. D. Serot, and H.-B. Tang, Nucl. Phys. A615, 441 (1997).

[18] J. Delorme, M. Ericson, P. A. M. Guichon, and A. W. Thomas, Phys. Rev. C 61, 025202 (2000).

[19] P. A. M. Guichon, Phys. Lett. B 200, 235 (1988).
[20] T. Hatsuda, T. Kunihiro, and H. Shimzu, Phys. Rev. Lett. 82, 2840 (1999)

[21] E. Akmedov, Nucl. Phys. A500, 596 (1989).

[22] G. Chanfray and D. Davesne, Nucl. Phys. A646, 125 (1999). 